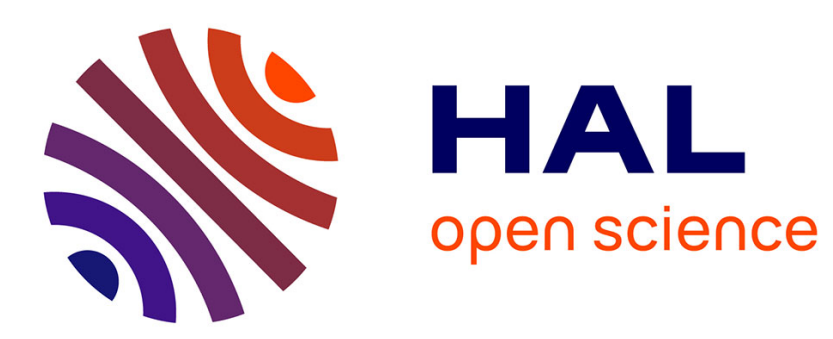

\title{
Reconstruction of deformed parent grains from microstructure inherited by phase transformations
}

Lionel Germain, Nathalie Gey, Michel Humbert

\section{To cite this version:}

Lionel Germain, Nathalie Gey, Michel Humbert. Reconstruction of deformed parent grains from microstructure inherited by phase transformations. Scripta Materialia, 2019, 158, pp.91-94. 10.1016/j.scriptamat.2018.08.042 . hal-02380651

\section{HAL Id: hal-02380651 \\ https://cnrs.hal.science/hal-02380651}

Submitted on 2 Dec 2019

HAL is a multi-disciplinary open access archive for the deposit and dissemination of scientific research documents, whether they are published or not. The documents may come from teaching and research institutions in France or abroad, or from public or private research centers.
L'archive ouverte pluridisciplinaire HAL, est destinée au dépôt et à la diffusion de documents scientifiques de niveau recherche, publiés ou non, émanant des établissements d'enseignement et de recherche français ou étrangers, des laboratoires publics ou privés. 


\title{
Reconstruction of deformed parent grains from microstructure inherited by phase transformations
}

\author{
Lionel Germain ${ }^{1,2^{*}}$, Nathalie Gey ${ }^{1,2}$, Michel Humbert ${ }^{1}$ \\ ${ }^{1}$ Université de Lorraine, CNRS, Arts et Métiers Paris Tech, LEM3, F-57000 Metz, France \\ ${ }^{2}$ Laboratory of Excellence on Design of Alloy Metals for low-mAss Structures (DAMAS), Université de \\ Lorraine, France
}

*Corresponding Author: lionel.germain@univ-lorraine.fr

\begin{abstract}
:
The methods used to reconstruct austenite grains from martensite measured by Electron Backscattered Diffraction rely on two assumptions: the orientation relationship and the parent orientation are unique locally. However in presence of an orientation gradient, the second assumption is no longer respected. Therefore in this work, we have first evaluated the deviation to both assumptions which revealed that the presence of an orientation gradient increases the deviation to the second assumption so that it becomes difficult to guaranty a reliable reconstruction. Therefore an adaptation of the method is proposed to better account for the orientation gradient and improve reconstruction results.
\end{abstract}

Keywords: EBSD; martensite; austenite; deformation; reconstruction

\section{Main article:}

Over the last decade, methods have been developed to reconstruct the parent microstructure (e.g., austenite) from EBSD measurements performed at room temperature on its transformation product (e.g., martensite, bainite...) [1-8]. The parent reconstructions offer great advantages over metallographic etching such as Bechet Beaujard [9] or thermal etching [10]. Indeed, their applications require only the acquisition of an EBSD map which is today a widely available characterization technique. Moreover, in addition to the parent grain structure, they also provide the individual orientations of the parent grains which may be used for instance to evaluate their crystallographic texture or to study the variant selection. Two different strategies have been used for fully automated reconstructions: Pixel by pixel reconstructions $[7,8]$ use the data at the pixel level whereas domain based reconstructions [1-6], use average orientations of crystallographic domains identified with a classical grain detection algorithm (e.g. as in [11]). The domain based reconstructions allows saving of computational time in the following steps because the number of domains is much lower than the number of pixels. However some information is lost during averaging which can be damageable when the orientation of the domain contain an orientation gradient like in deformed materials. In this article, the first part explains the assumptions on which domain based reconstructions relies. The second part assess the effect of a deviation to the assumptions. The third part describe briefly the reconstruction method and propose an adaptation to account for orientation gradient. In the last part an application example assess the benefit of the new method.

The domain based reconstruction methods are based on two assumptions. The first assumption is that at any location in the microstructure, a child orientation is related to its parent by a unique Orientation 
Relationship (OR). The second assumption is that the parent orientation is unique in a parent grain (or at least in a small neighborhood). Both assumptions have implication for the accuracy of a reconstructed map, especially when an orientation gradient existed before transformation. The objective of this paper is to evaluate the implication of each assumption and to propose an alternative method in the case of the presence of an orientation gradient when the second assumption is not respected anymore (e.g. in a deformed material).

In general, the relation between the orientation of a child crystal $g \alpha_{i}$ and its parent orientation $g \gamma$ is given by [12]:

$g \alpha_{i, j}=g \gamma \times P_{i} \times \Delta g \times C_{j}$

where $P_{i}$ are the rotational symmetry elements of the parent phase and $\Delta g$ is the OR expressed as a rotation. $C_{j}$ are the rotational symmetry elements of the child phase and only account for all equivalent rotations describing the same orientation. Conversely, all the potential parent orientations of a given child orientation are given by:

$g \gamma_{i, j}=g \alpha \times C_{i} \times \Delta g^{-1} \times P_{j}$

From the first assumption, $\Delta g$ is constant. Even if it was verified by an abundant literature for recrystallized [13] and deformed parent grains [14], it is important to assess how experimental data conform to this assumption. According to equation (1), an austenite grain with a single orientation transforms into several crystallographic variants, each with a single orientation. Any spread in a martensite lath orientation can therefore be attributed either to measurement errors or to a deviation to the first assumption. The GOS (Grain Orientation Spread) is a relevant metric of the orientation spread in EBSD maps [15]. This indicator is the average angular deviation of all points in a crystallographic domain with respect to its average orientation. Theoretically, this indicator is sensitive to low angle boundaries if the crystallographic domains are not properly defined. Here, to avoid any influence of sub-grain boundaries, the crystallographic domains were identified using a small tolerance angle of $3^{\circ}$ and sub-grain boundaries were closed using the ALGrld algorithm down to $1^{\circ}$ [16]. The Figure 1 compares the distribution of GOS for three metallurgical states: an austenite directly measured by EBSD on a stainless steel, a martensite formed from recrystallized austenite grains and an ausformed martensite. The GOS distribution in austenite quantifies directly the measurement error since there is no phase transformation. In general, it depends on measurement parameters but in standard EBSD conditions, the GOS is generally around $0.5^{\circ}$. Then in the martensite formed in a recrystallized austenite, all GOS values above $0.5^{\circ}$ characterize the deviation to the first assumption. The GOS distribution is shifted toward higher values with a peak at $1.5^{\circ}$ and a tail reaching $4^{\circ}$ as confirmed in [17]. This distribution explains why most reconstruction methods use tolerance angles around $3^{\circ}$ or above. However, it also shows that it shall not be necessary to use tolerance above $4^{\circ}$ if the $\mathrm{OR}$ is adequately determined. The data about the ausformed martensite shows that in the presence of an orientation gradient the peak in the GOS distribution stays at $1.5^{\circ}$ and the tail is shifted toward higher values.

The second assumption considers that the parent orientation is unique locally. This allows reformulating equation 1 and 2 as follows [18]:

$$
\left(g \gamma_{i, j}\right)^{-1} \times g \gamma_{k, l}=P_{i} \times \Delta g \times C_{j} \times g \alpha_{1}^{-1} \times g \alpha_{2} \times C_{k}^{-1} \times \Delta g^{-1} \times P_{l}^{-1}
$$


This equation equals the Identity when the two orientations $g \alpha_{1}$ and $g \alpha_{2}$ are strictly related to the same parent by the same OR. Any deviation the assumptions (or measurement error) results in a residual rotation of minimum angle $\theta$ which can therefore be used to evaluate how the reconstruction method is affected in the presence of an orientation gradient. Here a synthetic example as been designed so that only the second assumption shall influence the results. An austenite grain of $120 \mathrm{x}$ 120 pixels was created with an orientation $\left(g \gamma=\left[\phi_{1}: 107^{\circ} ; \Phi: 31^{\circ} ; \phi_{2}: 2^{\circ}\right]\right)$. Then a gradient was introduced by rotating all pixels of the grains depending on their position $(x, y)$ relative to the center of the grain. The rotation angle was $0.3^{\circ}$ per unit distance from the grain center (measured in pixels) and the rotation axis was calculated as $\vec{u}=\left(\frac{x}{60} ; \frac{y}{60} ; \sqrt{1-x^{2}-y^{2}}\right)$. The initial orientation was chosen because it appears as white in the Inverse Pole Figure color key with respect to $Z$ and reveals clearly the orientation gradient (Fig. 2a). The resulting Grain Orientation Spread (GOS) [15] is $13^{\circ}$ which is representative of a highly deformed grain. Then a martensite transformation was simulated by choosing a random child number (i.e., $i$ in eq. (1)). Then the trace of the corresponding (111) habit plane $\overrightarrow{v_{H P}}$ on the EBSD map was evaluated. Finally, all pixels lying at a distance of 3 pixels from a line parallel to $\overrightarrow{v_{H P}}$ crossing a pixel chosen at random underwent the $\gamma \rightarrow \alpha$ transformation according to equation 1 . The process was repeated until all pixels were transformed into martensite according to the Greninger Troiano (GT) OR. A cleaning step removed single pixel grains and replaced them by the orientation of one of their neighbors. The result is presented as an IPF map in Fig. 2c.

In domain reconstruction methods, the first step consists in detecting the domains and averaging their orientation. This step performed for the synthetic example is displayed in Fig. $2 \mathrm{~d}$. Then to evaluate the deviation to the second assumption at this step, the minimum rotation angle $\theta$ was evaluate and is displayed in Fig. $2 \mathrm{e}$ and $2 \mathrm{f}$ using a rainbow scale. The corresponding distributions are plot in the graph of Fig. 2g.

For the initial data (Fig. 2e), the $\theta$ distribution has a sharp peak at $0.3^{\circ}$ because the deviation is only inherited from the orientation gradient of the parent grain at the pixel scale (Fig. 2a). The deviation is much higher for the averaged data (Fig. 1f). In average, they are of $2.8^{\circ}$ with a tail reaching $11.8^{\circ}$. Fig. $1 \mathrm{~h}$ shows that longer domains have the highest deviations. For those domains, the deviation is close to the maximal GOS before averaging. The largest domain has a GOS of $9^{\circ}$ which corresponds to the distribution tail in Fig. 1g. Consequently, the tolerance used to find the parent must be increased by the same magnitude as the GOS induced by the gradient in the parent phase but on the length scale of the child domains.

In the original method [1], several domains are used and the most probable parent is the one related to most domains within a tolerance angle. Here, we have shown that in presence of an orientation gradient, this method is not optimal and therefore an adaptation is proposed. In equation (3), there are two ways to compute the misorientation between domains: the misorientation between average domain orientations ${\overline{g \alpha_{A}}}^{-1} \times \overline{g \alpha_{B}}$ which would be equivalent to the original method. Another way is to average the misorientation between domains: $\overline{g \alpha_{A}{ }^{-1} \times g \alpha_{B}}$. Then the most probable parent, is the one which minimize the tolerance angle $\theta$ with respect to all considered domains among all potential parents. This has the advantage that the tolerance should be increased only by a value equivalent to the orientation gradient between two pixels $\left(0.3^{\circ}\right.$ in the numerical example). 
The effect of the proposed adaptation (here after called gradient adaptation) was tested on an austenized and quenched $(A+Q)$ microstructure and compared to an austenized, rolled and quenched $(A+R+Q)$ one. Two low carbon steel samples $(0.2 \mathrm{C}-2 \mathrm{Mn}-2 \mathrm{Cr}$ wt\%) were used. The first one was austenized for $10 \mathrm{~min}$ at $850{ }^{\circ} \mathrm{C}$ and water quenched. The second was austenized at $950{ }^{\circ} \mathrm{C}$ for $30 \mathrm{~min}$, taken out of the furnace, $40 \%$ hot rolled and water quenched. At the end of hot rolling, the sheet temperature had decreased to $600{ }^{\circ} \mathrm{C}$ which implies that $5-10 \%$ of ferrite had formed before the transformation to martensite occurred. The samples were then ground to $1 / 4$ thickness and electropolished. Three EBSD maps were acquired for each sample using a Jeol JSM-7001F equipped with a Nordlys $S$ camera from Oxford Instruments. For the $A+Q$ condition, each map covered a surface of $130 \times 100 \mu \mathrm{m}^{2}$ with a step size of $0.1 \mu \mathrm{m}$. For the $A+R+Q$ condition, the microstructure was finer so a step size of $0.05 \mu \mathrm{m}$ was used, resulting in an analyzed surface area $85 \times 65 \mu \mathrm{m}^{2}$. One of the three IPF maps is displayed in Fig. 3a-b for both conditions; the sets of three maps were similar to each other.

Both the original and the gradient-adapted method were implemented in our reconstruction software Merengue 2 which is based on the algorithm described here [1]. To avoid any influence of the reconstruction parameters, a set of optimal parameters was determined by trial and error and kept constant for all reconstructions. One important parameter is the tolerance angle which was set to $3^{\circ}$ in all cases. Another important parameter is the OR. Two different methods have been used for the original and gradient adapted methods with the aim that both the determination of the OR and the reconstruction method relies on the same assumptions. As the original method uses average domain orientations, the OR was measured with a method based on average domain orientations [19] and only for the A+Q condition. Here the measured OR was $\Delta g=\left[\phi_{1}: 3.42^{\circ} ; \Phi: 45.9^{\circ} ; \phi_{2}: 5.97^{\circ}\right]$. In the case of the gradient adapted method, only misorientations are used in the reconstruction and an estimation of the OR must be made accordingly (also based on average misorientations between domains). For this purpose, the OR was measured using an accelerated version of the method described in [18] with the difference that the application was not made at triple junction but used rather averaged disorientations along boundaries. The measured $O R$ for $A+Q$ and $A+R+Q$ conditions differed only by $a$ disorientation angle of $0.2^{\circ}$; accordingly, the average OR of both conditions was used, $\Delta g=\left[\phi_{1}: 4.2^{\circ}\right.$; Ф: $\left.46.2^{\circ} ; \phi_{2}: 5.5^{\circ}\right]$.

The resulting reconstructions are presented in Figs. 3c-f. For the $A+Q$ condition, no orientation gradient is expected and both methods gave a high reconstruction fraction. The main difference concerns the shape of the twins which are more realistic with the original method. For the $A+R+Q$ condition, the original method allowed the reconstruction of only $50.8 \%$ of the map whereas the gradient adaptation increased this fraction to $70.8 \%$. If one accounts also for the presence of $5-10 \%$ ferrite in this microstructure, this gives a true reconstruction fraction between 74 and $82 \%$.

One of the reasons why the original method is somewhat better in recrystallized microstructure is linked to the first assumption. As already mentioned, not all pixels are related to their parent orientation by the same unique OR. For example, it was shown by Sato \& Zaefferer that orientation gradients exist in butterfly martensite as a result of the transformation of undeformed austenite [17]. Within each martensite lath, they observed that the OR varied from GT to KS. In a gradient-free reconstruction, one could account for these local variations by adapting the OR. Therefore, an estimate of the OR had been made according to the data used in the reconstruction (averaged domains orientation or averaged misorientations). Reconstructions made without an adapted OR resulted in a degradation of the results. Another factor may also influence the results. As shown by Wright et al., the angular resolution of EBSD is lower at grain boundaries [20]. Hence, the gradient -adapted method 
should be more sensitive to this phenomenon. By contrast, if the orientation gradient in the parent phase is large enough, the gradient-adapted method provides a significant improvement of the results.

In conclusion, the choice of the reconstruction method to be used depends on the gap with respect to the two assumptions. If the second assumption (that the parent orientation is unique) is applicable, the original method gives better results. If the deviation from this assumption becomes greater than the deviation from the first assumption (i.e. unique OR), the gradient-adapted method becomes more successful. The choice of the method can be done based on the prior knowledge of the thermomechanical treatment of the material or by using both method and retaining the one which give the better results.

This research was funded by the French State through the program "Investment in the future" operated by the National Research Agency (ANR) and referenced by ANR-11-LABX-0008-01 (Laboratory of Excellence 'DAMAS': Design of Alloy Metals for low-mAss Structures).

[1] L. Germain, N. Gey, R. Mercier, P. Blaineau, M. Humbert, Acta Mater. 60 (2012) 4551-4562.

[2] C. Cayron, J. Appl. Crystallogr. 40 (2007) 1183-1188.

[3] M. Abbasi, D.-I. Kim, T.W. Nelson, M. Abbasi, Mater. Charact. 95 (2014) 219-231.

[4] V. Tari, A.D. Rollett, H. Beladi, J. Appl. Crystallogr. 46 (2013) 210-215.

[5] A.H. Pham, T. Ohba, S. Morito, T. Hayashi, Mater. Charact. 132 (2017) 108-118.

[6] L. Sanz, B. Pereda, B. López, Metall. Mater. Trans. A 48 (2017) 5258-5272.

[7] G. Miyamoto, N. Iwata, N. Takayama, T. Furuhara, Acta Mater. 58 (2010) 6393-6403.

[8] N. Bernier, L. Bracke, L. Malet, S. Godet, Mater. Charact. 89 (2014) 23-32.

[9] S. Béchet, L. Beaujard, Rev. Métallurgie 10 (1955) 1045-1052.

[10] C. García de Andrés, F.G. Caballero, C. Capdevila, D. San Martín, Mater. Charact. 49 (2002) 121127.

[11] F. Bachmann, R. Hielscher, H. Schaeben, Ultramicroscopy 111 (2011) 1720-1733.

[12] M. Humbert, H. Moustahfid, F. Wagner, M.J. Philippe, Scr. Metall. Mater. 30 (1994) 377-382.

[13] A.-F. Gourgues, H.M. Flower, T.C. Lindley, Mater. Sci. Technol. 16 (2000) 26-40.

[14] S. Lubin, A.F. Gourgues-Lorenzon, B. Bacroix, H. Réglé, F. Montheillet, Solid State Phenom. 172174 (2011) 772-777.

[15] S.I. Wright, M.M. Nowell, D.P. Field, Microsc. Microanal. 17 (2011) 316-329.

[16] L. Germain, D. Kratsch, M. Salib, N. Gey, Mater. Charact. 98 (2014) 66-72.

[17] H. Sato, S. Zaefferer, Acta Mater. 57 (2009) 1931-1937.

[18] M. Humbert, L. Germain, N. Gey, E. Boucard, Acta Mater. 82 (2015) 137-144.

[19] M. Humbert, P. Blaineau, L. Germain, N. Gey, Scr. Mater. 64 (2011) 114-117.

[20] P.S. Bate, R.D. Knutsen, I. Brough, F.J. Humphreys, J. Microsc. 220 (2005) 36-46. 


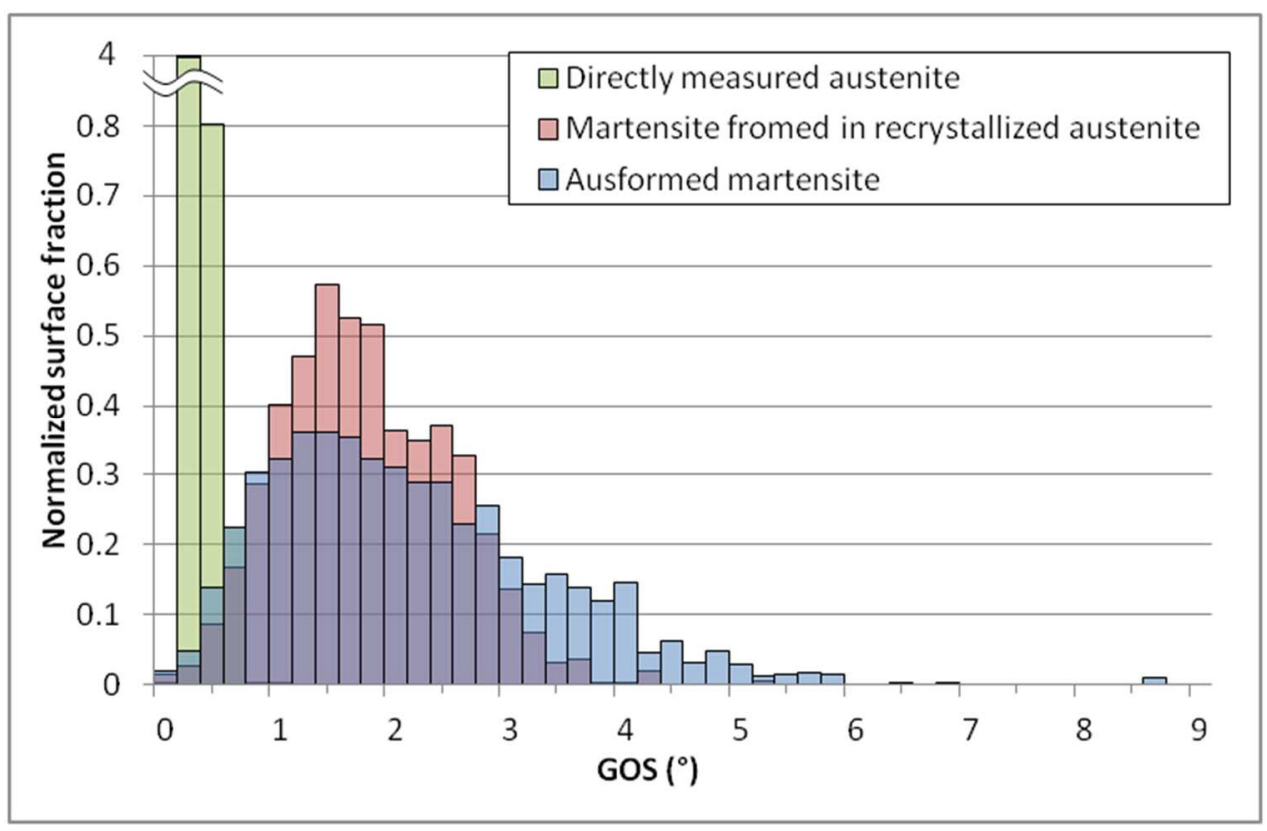

Figure 1: GOS distribution for three different metallurgical states. The crystallographic domains have been identified so that sub-grain boundaries do not influence the GOS distribution. 

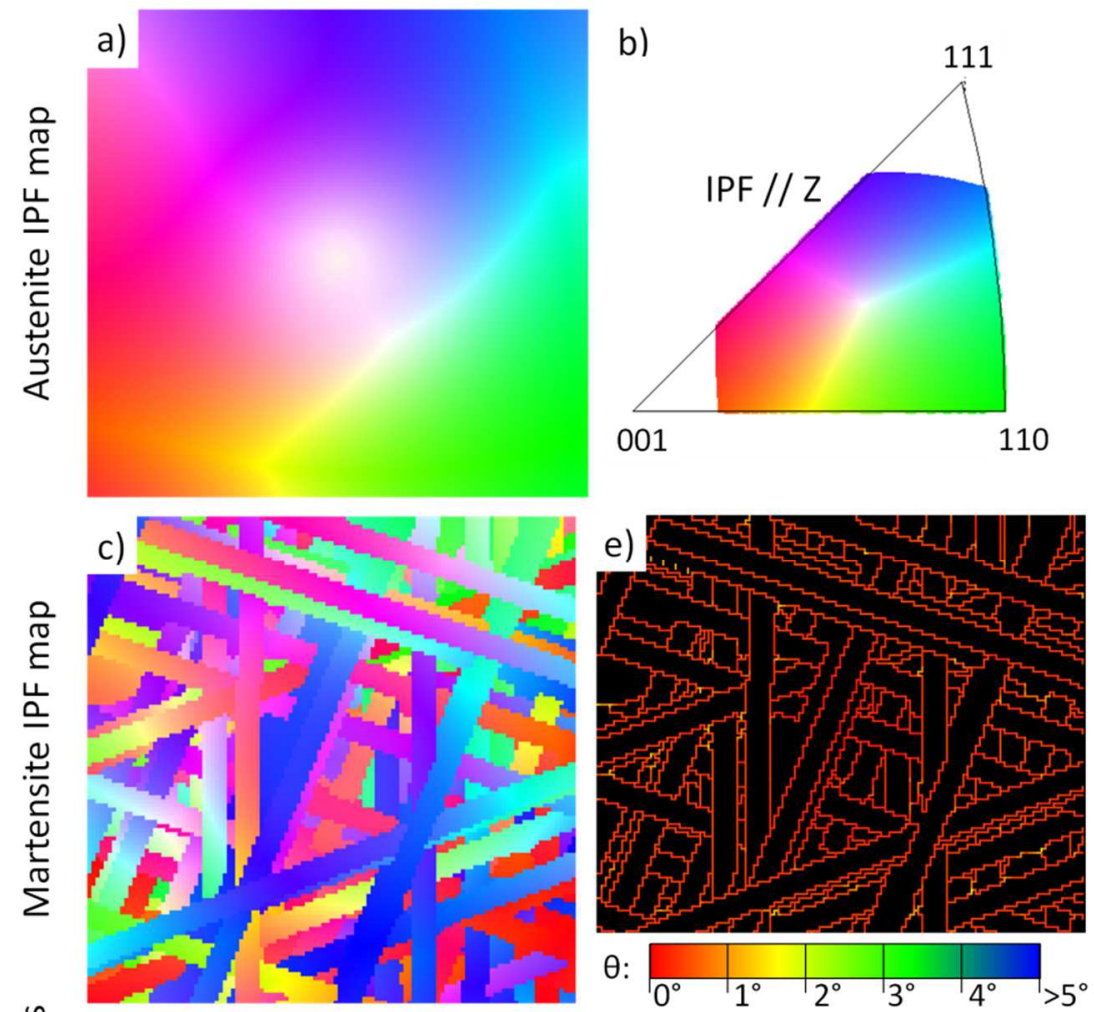

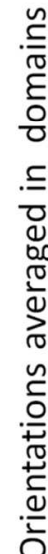
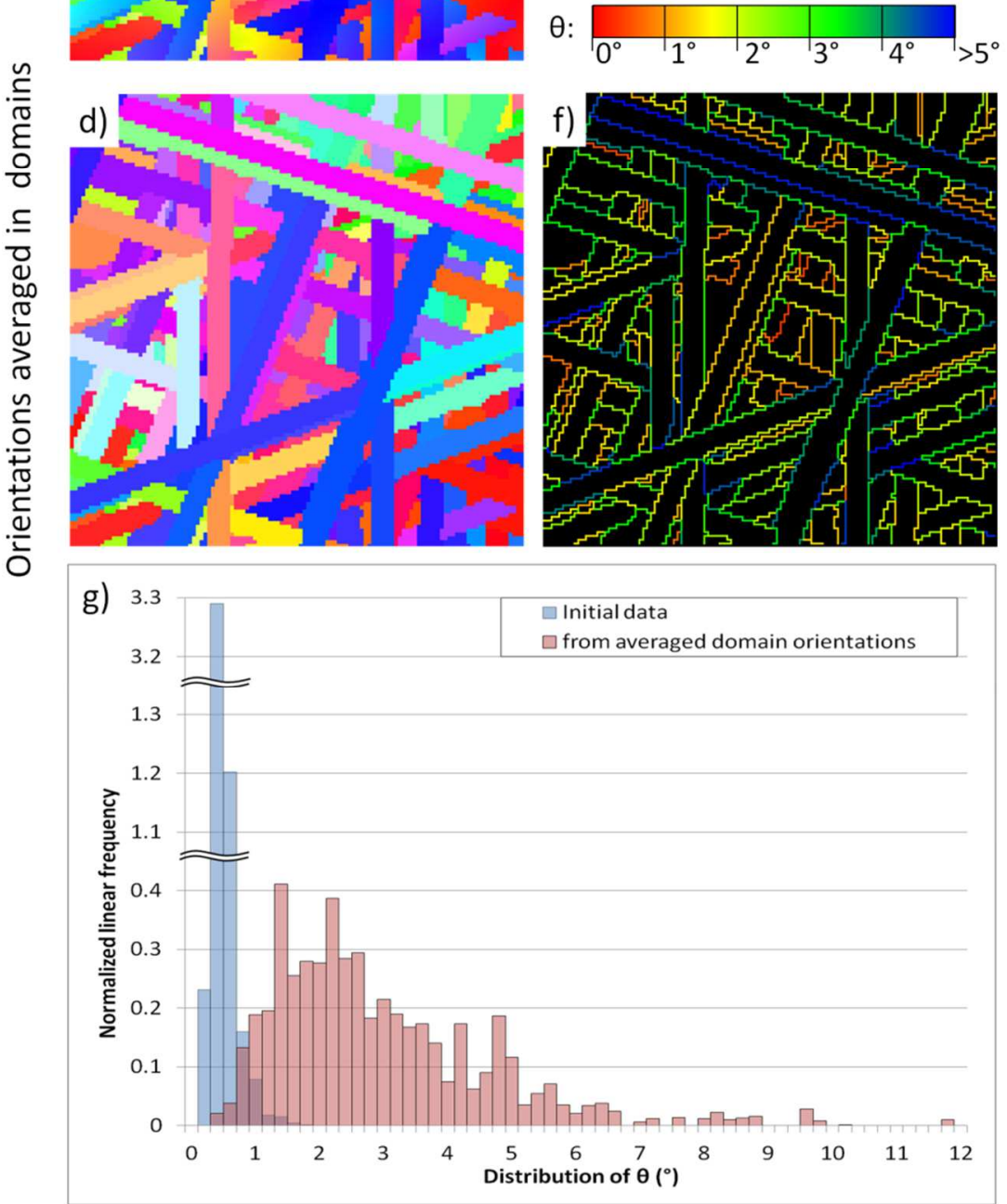

Figure 2: a) IPF map of a $120 \times 120$ pixel austenite grain with a $0.3 \%$ pixel orientation gradient. b) Inverse pole figure with respect to direction $\mathrm{Z}$. c) Simulated martensite microstructure where each pixel has inherited the orientation gradient of the austenite grain through eq. (1) d) data of figure $c$ but with orientations averaged over crystallographic domains, e-f) same figure as c-d) with GB colored to highlight their deviation to (eq. 3), g) distributions of the deviations to eq. 3. 

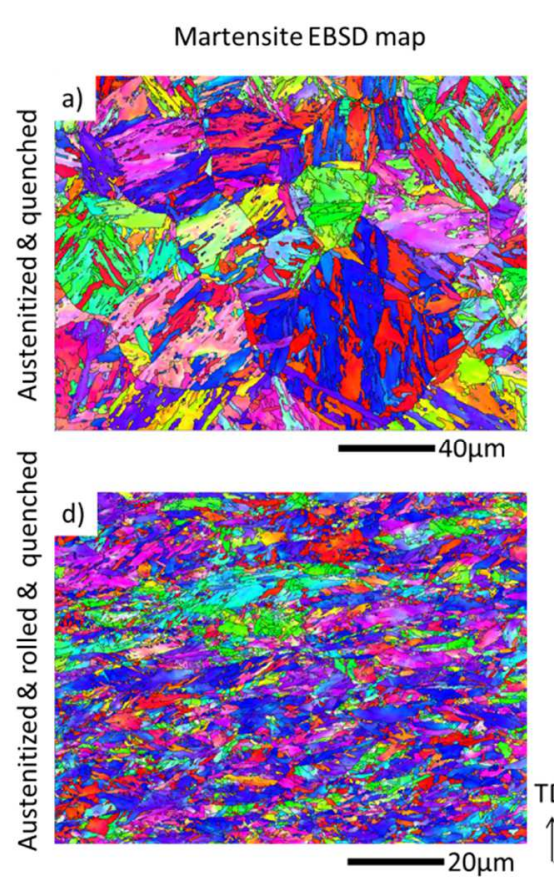

Austenite reconstructed with the classical domain method

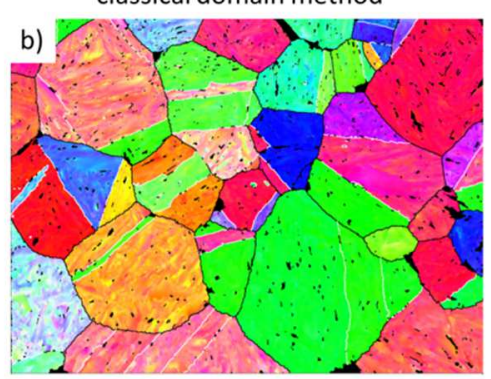

Austenite reconstructed with the

adapted domain method
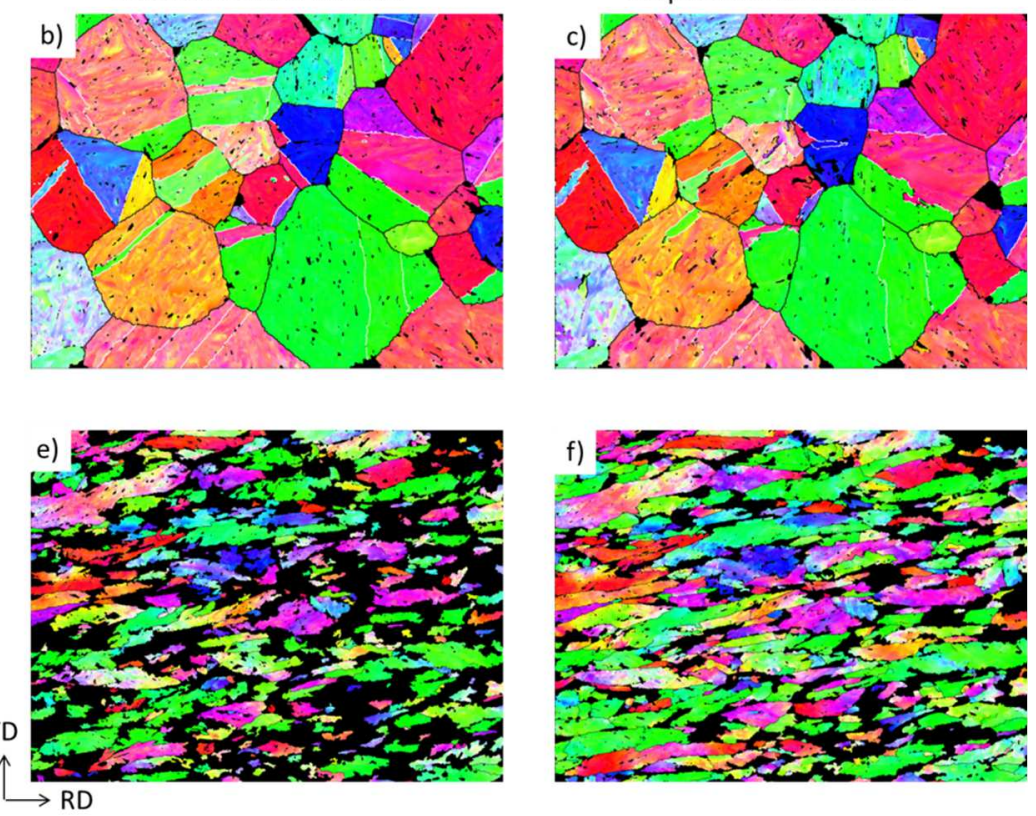

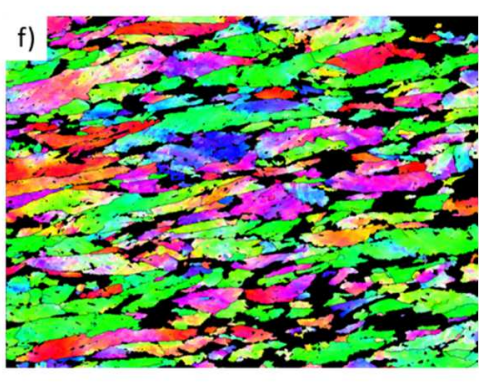

Figure 3: a) Measured EBSD map of the $A+Q$ sample, b) reconstruction with the classical method, c) reconstruction with the adaptation to orientation gradients, $d-f)$ same for the $A+R+Q$ EBSD map. 